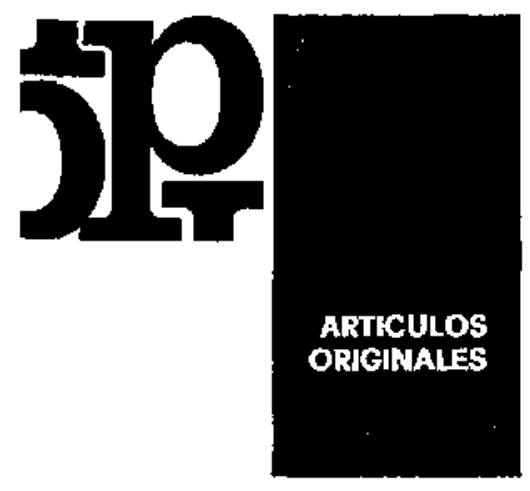

\title{
Control inmunológico del lupus eritematoso sistémico en 5 pacientes en edad pediátrica
}

\author{
DRS.: R. SORENSEN ***, M. MELENDEZ *, A. GALLETTI **, H. DEL POZO *** y F. PUGA ****.
}

INTRODUCción. Los hallazgos serológicos en pacientes con lupus eritematoso sistémico (LES) se caracterizan por un espectro sorprendentemente grande de fenómenos de autoinmunidad y por las consecuencias de la interacción de antígenos y anticuerpos (1).

En la patogenia de la enfermedad hay que considerar la acción directa de los autoanticuerpos sobre antígenos fijos y la acción indirecta a travês del depósito de complejos antígenos anticuerpos.

La mayoría de las lesiones en pacientes con LES, son inducidas por la acción de anticuerpos que son responsables de las alteraciones vasculares, principal Iesión anatomopatológica del LES. Esta lesión se caracteriza histológicamente por un engrosamiento de la membrama basal, la que inmunohistoquímicamente se caracteriza por el depósito de inmunoglobulinas, tercer factor del complemento (C3) y fibrinógeno (2) (3) (4). Es muy probable que este tipo de lesión vascular sea el resultado del depósito de los complejos antigenos anticuerpos en los vasos (5) (6) (7) (8). Los hallazgos inmunohistoquímicos son semejantes a la vasculitis de la enfermedad por complejo antígeno anticuerpo. (9) (10) (11) El compromiso predominante de algunos órganos se explica por las diferentes características de su red vascular que en algunos sitios favorece el depósito de complejos inmunes (1) (5) (9).

En esta comunicación presentamos nuestra experiencia en el control inmunológico de pacientes con LES. en los que se realizaron las siguientes determinaciones en forma seriada durante su evolución: inmunoglobulinas, factor 3 del comple-

\footnotetext{
* Sección Inmunopatologia, Instíluto Bacterjológico.

* Unidad de Jnmunologia, Hosp. L. Calvo Mackcnua.

*** Servicio Medicina Interna, Hosp. L. Calvo Mackenna.

** Departamento Nefrología, Hosp. L. Calvo Mackenna.
}

mento (C3), test de Coombs directo, anticuerpos antinucleares (AAN), recuento de linfocitos, junto a controles seriado de VHS., hemograma, y céhulas del lupus.

Material y MÉTODO. El material lo constituyen 5 pacientes del sexo femenino cuyas edades fluctúan entre los 9 años y los 16 años controladas en el policlínico de inmunología del Hospital $\mathrm{L}$. C. Mackenna, dos de ellas por un lapso superior a Jos 4 años.

La historia clínica y evolución del compromiso renal fueron analizados en otra publicación (12). Consideramos como signos de actividad clínica la fiebre, manifestaciones renales, articulares, neurológicas, psicoticas, cutáneas o hematológicas (13) (14).

Las determinaciones de inmunoglobulinas y $\mathrm{C} 3$ se realizaron según la técnica de inmunodifusión radial (15). Los resultados de las determinaciones de inmunoglobulinas fueron expresado e unidades internacionales según las recomendaciones de la O.M.S. (16) siendo los valores normales para IgG 11 8-204, IgM 133-185, UI. OMS × ml. El valor de $\mathrm{C} 3$ está expuesto en $\mathrm{mg}$.\% (normal 150 $\pm 50)$. Los AAN se determinaron por iamunofluorescencia usando como antígeno núcleos de glóbulos rojos de pollo (17), informándose títulos encontrados. Consideramos como patológicos aquellos sobre 1: 32.

El tratamiento se realizó con corticoides. En los períodos de actividad se aumentó la dosis a la necesaria para dominar las manifestaciones clínicas; logrado este objetivo se disminuye la dosis de esteroides a la mínima necesaria para que no reaparezcan signos de actividad. Sólo en este momento empleamos un esquema discontinuo cada 48 horas (18) (19) (20). 


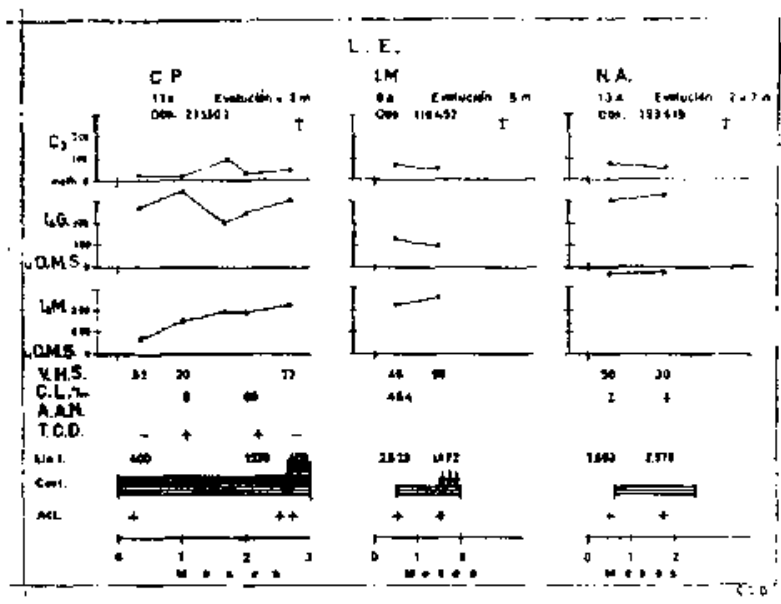

Figura Nọ 1.- Evolucion de tres casos de LES con hiprocomple:mentemia mantedicla.

Resultados. En la figura No 1 resumimos la evolución de tres pacientes, a uno de los cuales (Obs. 213602) no se realizo biopsia renal (14).

C. P. (Obs. 213602). Paciente de 11 años de edad cuya evolución fue fatal a los 8 meses de iniciadas sus primeras manifestaciones y a los tres meses de diagnosticada. Las manifestaciones clinicas de actividad, salvo un breve periodo, fueron permanentes, acompañadas con niveles séricos de C3 muy descendidos e inmunoglobulinas $G$ elevadas. La linfopenia fue persistente. La respuesta al corticoides fue mala, la causa de muerte fuc una neumonia.

I. M. (Obs. 16432). Paciente de nucve años de edad que fallece al cabo de 5 meses de evolución. Su actividad clínica y niveles bajos de C3 son persistentes, la linfopenia es moderada. Por la mala respuesta esteroidal se asocia un inmunosupresor que le produce una aplasia medular, causa inmediata de su muerte.

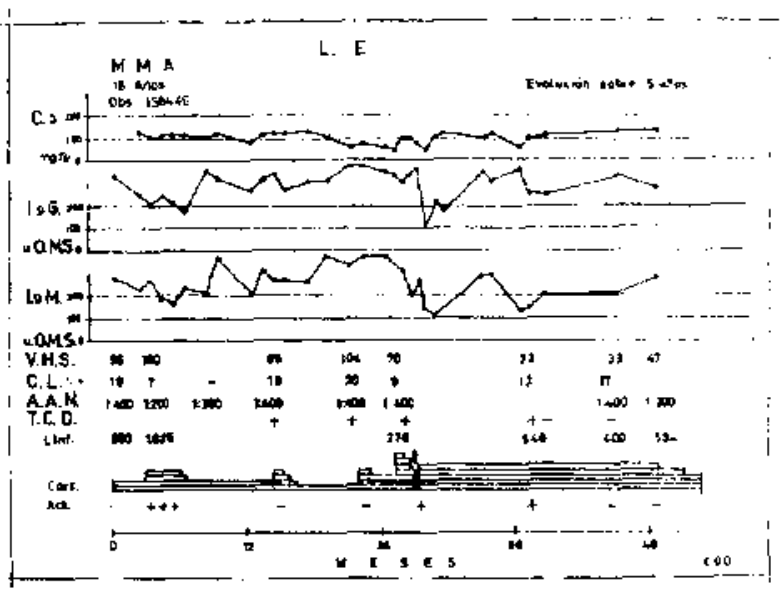

Figura No 2.- Evolución de un caso de LES sin bipoconple. mentemia mantenida.
N. A. (Obs. 193619). Paciente de trece años de edad que fallece a los 2 años 7 meses de evolución. Su actividad clínica fue persistente, con niveles séricos de $\mathrm{C} 3$ bajo e inmunoglobulina 0 elevada. La linfopenia fue leve. Fue resistente el tratamiento esteroidal.

En la figura No 2 se resune la evolución de M. M. A. (Ohs. 156446), paciente cuyas manifestaciones iniciadus el año 1966 indujeron al diagnóstico de artritis reumatoídea tratándose como tal durante 1 año 4 meses. EI año 1968 se diagnosticó LES iniciándose tratamiento.

Inicia sus controles inmunológicos en Marzo 1969 en momentos en que estaba recibiendo $30 \mathrm{mg}$. de corticoides diarias.

En Mayo de 1969 hay marcados signos de actividad: anasarca, hematuria y proteinuria. El C3 está en límite normal bajo y la inmunoglobulina $G$. elevada, los AAN tienen títulos elevados. Hay además VHS elevada y linfopenia moderada. Por estas alteraciones se aumenta la dosis de corticoides a $50 \mathrm{mg}$. diarios con buenos resultados.

En Mayo 1970 , estando con $20 \mathrm{mg}$. diarios de prednisona y asintomática, se pesquisan valores de C3 bajo lo normal unido a elevación de la VHS, células del lupus (CL) y títulos elevados de AAN. Estas alteraciones, pese a estar asintomática, inducen a aumentar la dosis de corticoides a 50 mgs. diarios para luego de acuerdo a la cvolución disminuir a 20 mgs. diarios.

En Noviembre 1970 los parámetros hematológicos están francamente alterados, estando asintomática, por lo que se aumenta la dosis de prednisona a $50 \mathrm{mg}$. diarios. Es importante destacar que a estas alturas de la evolución las dosis de corticoides fueron ajustadas inmediatamente al producirse las alteraciones serológićcts sin esperar signos de actividad clínica.

En Abril 1971 pese a estar asintomática, los parămetros inmunológicos persisten alterađos, lo

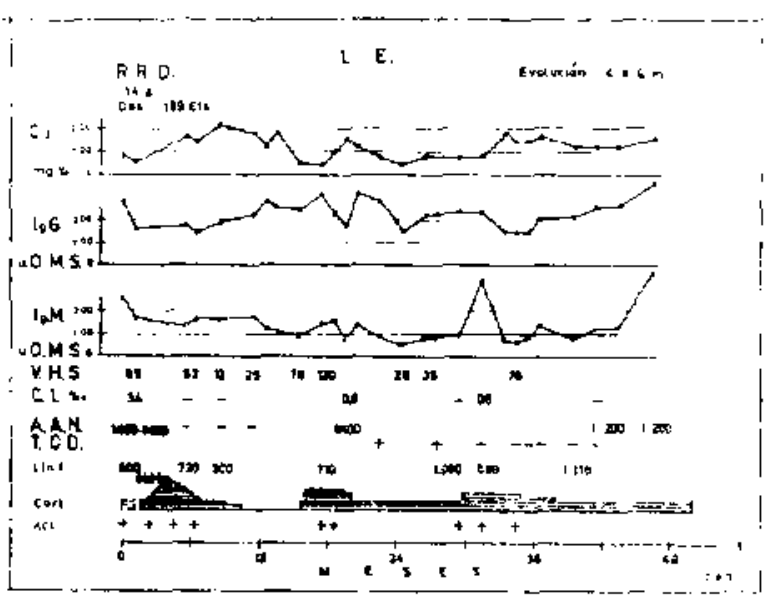

Figuran No 3.- Evolución de un coso de LES sim hipocotoplementenencia mantenida. 
que obliga a aumentar la dosis de prednisona a 80 mg. diarios. En momentos en que se estaba disminuyendo la dosis, éste se debe reemplazar por ciclofosfamida $1.5 \mathrm{mg} x$ peso diarios, por lo presencia de una úlcera corneal herpética, lo que se tradujo en una baja acentuada de $\mathrm{C3}$ seguido de una reactivación clínica. Dos semanas después se reinicia tratamiento esteroidal con una dosis de $60 \mathrm{mg}$. diarios logrando llevar al C3 a niveles normales junto a una revisión clínica.

La evaluación posterior ha sido satisfactoria a excepción de una oportunidad en Abril de 1972 en que hay baja de C3 y alteración de otros parámetros de laboratorio coincidiendo con un eritema facial intenso.

En la actualidad está asintomática y la dosis de corticoides se está disminuyendo en forma progresiva, bajo permanente control inmunológico.

El compromiso renal de esta paciente fue pesquisado precozmente y su evolución ha sido bastante satisfactoria.

La figura 3 resume la evolución de R. R. D. cuyas manifestaciones se inician en Noviembre del año 1968. Cinco meses después ingresa al Hospital Luis Calvo Mackenna, donde se diagnosticó LES.

Su control inmunológico se inicia en Abril 1969 estando clínicamente activa con niveles muy bajos de C3 acompañados de elevación de inmunoglobulinas y títulos de AAN. No se aumentó la dosis de prednisona por un atraso en su control.

Un mes después, en Mayo 1969, se planteó el diagnóstico de peritonitis secundaria a una biopsia renal. Considerando que el corticoides estuviese enmascarando el cuadro se suspende, reactivándose la fjebre a tal punto que obliga a reiniciar tratamientos esta vez utilizando Betametazona por presentar una gastroentiritis aguda. En Junio de 1969 la enferma se encuentra en estado grave con un gran número de manifestaciones de LES: compromiso cutáneo, hematológico, meningoencefalitis, alteraciones del comportamiento, pleuritis, insuficiencia renal aguda y además sepsis y diarrea. Junto al tratamiento hidroelectrodílico $\mathrm{y}$ químioterápico, ante la magnitud del cuadro y la persistencia del $\mathrm{C} 3$ descendido con aumento del título de AAN, se decide a aumentar la dosis de Betametazona y asociarla a prednisona llegando esta última de $180 \mathrm{mg}$. diarios. Posteriormente los parámetros inmunológicos mejoran al igual que las manifestaciones clínicas de tal manera que permite la disminución de la prednisona hasta llegar a 10 mg. diarios.

Con posterioridad volvió a presentar varias recaídas, siempre precedidas por un descenso de C3. Cada vez se lograron controlar ajustando la dosis de corticoides.

$7 \%$

Comentakjo. El análisis de esa casuística permiten separar entre 3 casos que fallecieron luego de una corta evolución (1) con hipocomplementemia persistente a pesar de la terapia estecoidal en 2 pacientes (CP y NA) y de tratamiento combinado con un inmunodepresor en el otro paciente (IM). Los otros 2 casos (M. M. y R. R.) corresponden a pacientes sin hipocomplementemia mantenida, guardando el descenso de los valores séricos de C3 estrecha relación con períodos de actividad clínica o laboratorio. La normalización de C3 se tradujo en una remisión, producto de la buena respuesta a una dosis adecuada de esteroides. Diversas publicaciones (21) (22) (23) (24) (25) (26) (27) coinciden en la utilidad de las determinaciones seriadas de esta proteína sérica en el manejo terapéutico LES, observaciones que se cumplen en todos nucstros pacientes en el sentido de que el descenso de C3 nunca dejó de ser seguido por una reactivación del cuadro clínico en aquellos casos que no se ajuste la dosis de prednisona de acuerdo a este hallazgo y hasta lograr un retorno de $\mathrm{C3}$ a niveles normales.

E1 descenso de C3 sérico es más intenso en pacientes con marcado compromiso renal que en aquellos sin este compromiso (21) (22). El consumo de C3 en pacientes con LES coincide con la presencia de complejos antígenos-anticuerpos circulantes (28) (6).

En estos pacientes se efectuó en forma rutinaria la determinación de niveles de C3. Este componente del complemento es el que se encuentra en mayor concentración en el suero y su consumo se evidencia de una activación importante del sistema del complemento. En algunos se ha visto atle existe una disminución de la capacidad hemolizante del complemento sin descenso de niveles de $C 3$, debido a que se consume antes $\mathrm{C} 2$ cuya inactivación hace bajar la capacidad hemolítica global del complemento, pero no necesarjamente produce una disminución de C3. En los casos de duda conviene efectuar ademâs la determinación de la actividad hemolítica del complemento en suero fresco (28).

Los valores de inmunoglobulinas sufren variaciones importantes ducante la evolución, pero no hay una relación entre la variación de sus niveles y actividad de la enfermedad (27). La no elevación de la IgG en un caso podría explicarse por una importante disminución de la vida media de las inmunoglobulinas observado en esta enfermedad (29). Algunos autores encuentran una relación entre la elevación de inmunoglobulinas y consumo de $C(27)$, hecho que no podemos confirmar observando muestro material. Por el contrario. en 3 de nuestros pacientes (Y. M., M. M. y R. R.) el consumo de G3 coincidió con una caída importante de los niveles de IgG e IgM. lo cual podría ser interpretado como una activación del catabolismo de las inmunoglobulinas. Esto tiede importancia clínica, pues no debe esperarse en- 
contrar siempre un aumento de gamaglobulina (que corresponde fondamentalmente a $\mathrm{IgG}$ ) para aceptar el diagnóstico de LES.

Las células del LES o células de Hargraves fueron pesquisadas en nuestros pacientes encontrándose presentes en todos ellos. Los porcentajes encontrados son mucho menores que los que se describen en adultos en que se requiere un mínimo de $2 \%$ de células para plantear el diagnóstico de LES.

La formación de células LE es inducida por la presencia de un anticuerpo antinuclear que actúa sobre los núcleos de leucocitos que han sido dañados levemente en vitro al desfibrinar la sangre. Esto permite que los anticuerpos lleguen al núcleo y desencadenan un mecanismo en el cual probablemente una desoxiribonucleasa destruye las cadenas polipeptídicas que constituyen la estructura de las nucleoproteínas, produciendo una masa totalmente amorfa que luego es fagocitada por un leucocito intacto. Este fenómeno típico debe diferenciarse de la núcleofagocitosis, fenómeno que se presenta en procesos como sepsis, etc. y que se diferencia de la formación de células LE porque el material fagocitado no ha perdido su estructura. En nuestro material de niños pensamos que cuando el fenómeno de Hargraves es típico, tiene importancia diagnóstica aún cuando no tenga la intensidad oue presenta en adultos. (30) (31) (32) (33) (34).

En LES la determinación de AAN es de alto valor diagnóstico (30). Se encuentran aun en pacientes tratados en los cuales no se evidencia el fenómeno de Hargraves.

Nosotros empleamos una técnica de inmunofluorescencia utilizando como fuente de antígeno núcleos de GR de pollo (17). El rol de los AAN no es claro en la patogenesis del LES: de hecho por sí mismo no son patogénicos (36) (37) (38). Se ha demostrado la oresencia de ellos en Recién Nacidos hijos de madre con LES sin que signifiquen enfermedad para ellos. (39) (40) (41).

Probablemente la acción de este tipo de anticurerpo en vivo no se deba a un daño directo de los núcleos celulares, a los cuales no tienen acceso, sino a la formación de complejos antígenos-anticuerpo circulantes. El antígeno estaría dado por DNA o RNA libre en el suero. El daño del árbol vascular, en especial en el riñón y en los plexos coroides, se debe al depósito de estos complejos, con atracción subsecuente de complemento y luego leucocitos que liberan enzimas proteolíticos de sus lisozomas.

La presencia de AAN. se puede valorar tanto por la imagen de tinción que produce en el núcleo (42) como por su título (43) (44). Nosotros no damos gran importancia a la imagen observada, pues esta varía con la dilución del sueto que se estudia. El hecho fundamental para nosotros es el título en el cual se encuentran estos anticuerpos: hasta 1: 32 se encuentran en personas normales y hasta 1: 200 lo hemos observado en Artritis Reumatoídea, linfomas, etc. Título de 1: 400 o más en nuestra experiencia son altamente sugestivo de LES (45).

El AAN puede encontrarse en la fracción IgG, IgM y $\operatorname{IgA}$. (46) (47). Adquiere mayor importancia etiopatogénica cuando es capaz de captar complemento.

La linfopenia observada en algunos pacientes lúpicos puede deberse a varias causas: se han descrito anticuerpos antilinfocitarios en la fase de actividad de lupus, y por otra parte el tratamiento con corticoides lleva a linfopenia. Se han planteado que aún en estos pacientes podría existir una deficiencia de linfocitos timo dependientes como condicionante de la enfermedad.

En el tratamiento utilizamos esteroides (48) considerando que el efecto de esta droga es tanto más benéfica cuanto más precoz sea su administración.

Es importante el ajuste frecuente de la dosis de acuerdo a los hallazgos clínicos e inmunológicos, siendo de gran valor práctico la determinación de C3, y titulación de anticuerpos antinucleares. Al igual que lo comunicado por otros (20) (50) (51) en nuestros pacientes el resultado del tratamiento dependió fundamentalmente del grado del compromiso renal.

Sólo en dos de nuestros pacientes (M. M. y S. M.) utilizamos inmunodepresores. En un caso (IM) usamos Methotrexate en la fase final de la enfermedad, sin éxito. En el $2^{\circ}$ caso (M. M.) empleamos ciclofosfamida en un período en el cual debimos suspender los corticoides por una queratitis herpética. Con esta droga sola no logramos lievar a lo normal los niveles descendidos de C3.

\section{RESUMEN Y CONCLUSIONES}

La experiencia adquirida en el manejo clínico y de laboratorio de 5 casos de lupus comentados en este trabajo, nos permite destacar los siguientes hechos; 1) La determinación de varios parámetros inmunológicos, en especial de los niveles de complemento, en todos los controles de estos pacientes permite una regulación muy precisa de la dosis de corticoide (e inmunodepresores) requerido para evitar un daño progresivo.

2) Este tipo de evaluación clinica y de laboratorio nos ha permitido en la actualidad encontrar wn gran número de casos nuevos, diagnosticados en forma precoz y que están en este momento en control de nuestro Policlinico de Inminología. 


\section{SUMMARY}

The evolution of 5 cases of SLE is presented. All of them had serial determinations of $\mathrm{C} 3, \mathrm{I}_{\mathrm{gs}}$, and antinuclear factor. The close observation of these parameters mainly C3, allowed a frecuent ajusment of the required dosis of corticosteroids.

This tipe of clinical and laboratory evaluation has made it possible to detect several new cases in an early stage of the diseases.

\section{REFERENCIAS}

1.-Miescher, P. A. y Paronetto, F. Lupus Eritematoso Generalizado. En tratado de Inmunopatología. Dres. Miescher, PA., Müller-Eberhard, HJ. Edit. Científica Médica. Barcelona, 1971, II: 751.

2.-Paronetto, $F$, and Koffler, D. Inmunofluorescent localization of Inmunoglobulins, complement and fibrinogen in human diseases. I Systemic Lupus Erytematosus, J. Clin. Invest. 44: 1657, 1965.

3.-Svec, K., Blair, J. and Kaplam, M. Immunopathologic studies of Eystemic Lupus Erytematosus. I Tissue-bound immunoglobulins in relations to serum antinuclear Immunoglobulins in Systemic Lupus and in chronic liver diseases with $\mathbf{L E}$ cell factor. J. Clin. Invest. 46: 558, 67.

4.-Krishman, Ch., and Kaplan, M. Immunopathologic studies of Systemic Lupus Erythematosus. II Antinuclear reaction of gamma globulin eluted from homogenates and isolated glomeruli of kidneys from patients with Lupus nephritis. J. Clin. Invest. 46: $569,1967$.

5.-Atkins, C., Kondon, J., Quismorio, F. P. The choroid plexus in SLE. Ann. Int. Med. 76: 65, 1972.

6.-Galletti, A,, Sörensen, R. Mecanismos Inmunológicos en lesiones renales. Rev. Chil. Ped. 44: 73, 1973.

7.- Freedman, P., y Markowitz, A. S. Isolation of antibody-like gamma globulin from lupus glomeruli. Brit. Med. J. 1: 1175, 1962.

8.-Koffler, D., Schur, P. and Kunkel, G. Immunological studies concerning the nephritis of systemic lupus erytematosus. J. Exp. Med. 126: 607, 1967.

9.-Kaiker, W., und Cochrane, Ch. The localization of circulating complexes in experimental serum sickness. J. Exp. Med. 127: 119, 1968.

10.-Ward, P. A., and Cochrane, C. G. Bound complement and immunologic injury of blood vessels. $J$. Exp. Med. 121: 215, 1965.
11.-Dixon, F, J., Vásquez, J. J, Weigle, W. O., y Cochrane, C. G. The pathogenesis of serum sickness. A. M. A. Arch. Path. 65: 18, 1958.

12.-Galletti, A., Rosemberg, H., Allende, N., Sörensen, $R$., y Puga, F. Nefropatía del LES en la infancia. Rev. Chilena Pediatría, 44: 1, 1973.

13.-Meislin $A G$., Rothfield N.: Systemic Lupus Erytematosus in childhood. Pediatrics 43: 37, 1968.

14,- Cohhan AS., Reynolds, WE., Franklin, EC. et al. Preliminary criteria for the classification of systemic lupus erytematosus. Bull. Rheum. Dis. 21 : $643,1971$.

15.-Mancini, G., Carbonard, A. O., and Heremans, J. $F$. Immunochemical quantitation of antigens by single radial immunodifusión. Immunochem. 2: 235, 1965.

16.-Rowe, D. S., Anderson, S. G., Grab, B. A research standard for Human Serum Immunoglobulins IgG., IgA. and IgM. Bull. Org. Mond. Santé, 12: 535, 1970.

17.-Valdivieso $M$., Sthandiet $O$., Hurtado $R$. Titulación de anticuerpos antinucleares. Bol. lnst. Bact. Ch. 13: 21, 1971 .

18.-Dubois, E. L. SLE recent advances in its diagnosis and treatment. Ann. Int. Med. 45: 163, 1956.

19.-Ackerman, G. L. Alternate-day steroid therapy in Lupus nephritis. Ann. Int. Med. 72: 511, 1970.

20.-Danadio, J. V., Holley, K. E., Wagner, R. et at. Treatment of lupus nephritis with prednisone and combined prednisone and Azatioprine. Ann. Int. Med. 77: 829, 1972.

21.-Townes, A. S., Stewart, C. R., and Oster A. G. I Immunologic studies of systemic lupus erythema. tosus II Variations of nucleoprotein reactive gamma globulin and hemolytic serum complement levels with diseases activity. Bull Johns Hopkins Hosp. 112: 202, 1963.

22.- Tawnes, A. S., and Stew'ard, C. R. Estimations of the second component and third component complex of complement in the serum of patients with systemic Iupus erytematosus. Bull. Johns Hosp. 117: 348, 1965.

23.-Shur, Ph., Sandson, J. Immunologic factors and clinical activity in systemic lupus erythematosus. N. Eng. Med. 278: 533, 1968.

24.-Hanauer L. B., and Christian, Ch. L. Clinical StuStudies of hemolytic complement and the 118 component, Amer. J. Med. 42: 882, 1967. 
25.-Ellis, HA., and Davies, F. Serum complement, rheumatic factor and other serum proteins in rhematoid disease and SLE. Ann. Rheum. Dis, 18: $215,1959$.

26.-Willains, RC. and Law, DM. Serum complement in connect ive tissue disorders, J. Lab, Clin. Med. 52: $273,1958$.

27.- Vaughat, JH. Bayles TB, and Farour CB. The response of serum gamma globulin level and complement titer to adrenocorticotropic hormone (ACTH.) therapy in SLE. J. Lab. Clin. Med. 37 : $698,195 \mathrm{~J}$.

28.-Agnello, V. Koffler, D., Eisenberg, JW., et al. Clq precipitins in the seta of patients with SLE and other hypocomplementemic states: characterization of hight and low molecular weight tipes. J. Exp. Med. 1341: 228, 1971 .

29.-Levy J., Barnett, E. V., McDonald NB. and Klintemberg $J \boldsymbol{R}$. Altered immunoglobulin, metabolism in SLE and Rheumatoid arthritis. J, Clin. Invest. 49: $708,1970$.

30.- Weir DM., Holborow EJ. and Jolnson GD. A clinical study of serum antinuclear factor. Brit. $M$. J. $933: 5230,1961$.

31.- Lee SL., Michaet SR, and Vural $J$. The LE cell. Clinical and chemical studies. Amer. J. Med. 10: 446, 1951.

32.-Goodman HC. Fahey JL., Malmgren RA, and Brecher $\boldsymbol{G}$. Separation of factor in LE. serum reacting with components of cell nuclei. Lancet 2: 382 , 1959.

33.-Hargraves MM., Richmond $H$., and Morton $R$, Presentation of two bone marrow elements the "tart" cell and the LE cell. Proc. Mayo Clin, 23: $25,1948$.

34.-Hargraves MM., Richmond $H_{,}$, and Morton $R$. Production in vitro of the LE cell phenorenon. Proc. Mayo Clin. 24: 234, 1949.

35.- Circar F. y Farreras P. LES. En diagnóstico Hernatológico, laboratorio y clínica. Edit. Jims, Barcelona. 1972, p8. 956.

36.- Marmont AM. The transfusion of active LE plasma into non lupus recipients with a note on the LE like cel.l Ann. N. Y. Acad. Sci. 124, 838, 1965.
37.- Natall G., and Tan EM. Experimental renal disease induced by DNA-anti DNA inmune complexes. J. Cli Invest. 51; 345, 1972.

38.-Ward JR. Cloud RS, and Turrer LM. Non-cytotoxicity of nuclear antibodies from SLE sera in tissue cultare. Ann. Rheum. Dis. 23: 381, 1964.

39.--Beck JS., Oakley CL., anad Rowell NR. Transplacental passage of antinueclear antibody. Studies in infants of mother with SLE. Arch. Derm. 93: $656,1966$.

40.- Bridge $R G$, and Foley $F G$. Placental transmission of the LE. Am. J. M. Sc. 227: I, 1954.

41.-Berlyne GM., Siort LA. et al. Placental transmition of the SLE factor. Lancet 2: 15, 1957.

42.-Gonzale E., Rothfield $N$. Immunoglobulin slass and patiern of nuclear fluorescence in SLE. N. Eng. J. Med. 274: 1334, 1966.

43. - Ritchie $R$. The clinical significance of titered AAN. Arthritis Rheum, 10: 544, 1967.

44,- Mandeme E. Pollak VE., Kark RM., and Rezaian J. Quantitative observations on antinuclear factors in SLE. J. Lab. Clin. Med. 58: 337, 1961.

45.-Friou $G$. Antinuclear antibodies: diagnostic significance and methods. Arthritis Rheum. 10: 151, 1967.

46.- Barnett EV., North $\boldsymbol{A F}$, et al. Antinuclear antibidies in SLE and rheumatoid arthritis. Ann. Intern. Med. 63: 100, 1965 .

47.- Barnett EI., Condemi $J$. et al. Gamma 2, gamma AI and gamma IM antinuclear factors in human sera. J. Clin. Invest. 43; $1104,1964$.

48.- Lange $K$., Ores R. et al. Steroid therapy of SLE based on immunologic considerations. Arthritis Rheum. 8: 244, 1965.

49.- Pollak VC., Pirani C., Kark RM. Effect of large doses of prednisone on the renal lesions and life span of patients with lupus glomeralulonephritis. J, Lab. Clin. Med. 57: 495, 1961.

50.-Cameron JS., Boulion-Jones et al. Treatment of lupus nepbritis with cyclophosphamide. Lancet 2: $846,1970$. 